\title{
Application of Jurkevich Method in the Study of Optical Variability Period
}

\author{
Haiyan Yang ${ }^{1, a^{*}}$ and $\mathrm{Na}$ Jiang ${ }^{1, \mathrm{~b}}$ \\ ${ }^{1}$ School of Physics and Information Engineering, Zhaotong University, Zhaotong, China; \\ aelaincoco@foxmail.com, b27805044@qq.com
}

Keywords: Blazar; Jurkevich method; Variability period; Light curve

\begin{abstract}
Blazar is a highly active objects, which belongs to active galactic nuclei. Based on the statistical analysis of some Blazar objects in the literature, it is found that Jurkevich method is widely used in the study of astrophysics. The conclusions of much literature show that the Jurkevich method is a more accurate method on the study of the optical variation period of active galactic nuclei. Because of the low requirement and simple operation of Jurkevich method, it is also a very common method to analyze the active galactic nuclei.
\end{abstract}

\section{Introduction}

Astrophysical research has great influence on mankind, especially on human life. In the study of astrophysics, the study of active galactic nucleus has attracted wide attention. Active galactic nucleus, abbreviated as AGN, is a large class of extragalactic objects. Active galactic nucleus are characterized by nuclear activity, which is another source of energy produced in stellar thermonuclear reactions, and has a considerable impact in some phenomena. This definition is not quantitative. Since the nucleus of normal galaxies are also active, the lower boundaries of active galactic nucleus can extend to some normal galaxies, such as the Galactic center. The galaxies where active galactic nucleus host are known as active galaxies, according to habits, we also collectively consider less active active galaxies as active galactic nucleus.

Active galactic nucleus have all or part of the following observed characteristics: dense nucleus brighter than normal galaxies; continuous spectrum of radio, optical, X-ray and other wave bands with small bands is non-stars; there are atoms and ions in the emission spectrum; the intensity, polarization and shape of continuous spectrum and emission line change with the change of time; normal galaxies, such as $\gamma$ rays, have higher ability to transmit higher energy photon than X-ray do.

Mainly divided into: Seyfert galaxy, quasars, radio galaxies, BL Lac, optical cataclysmic quasars. Quasars: Active galaxies with the strongest activity; Seyfert galaxies: Quasars with brightness below - 24 (absolute magnitude); BL Lac: Active galaxies with optical variability and polarization; Radio galaxies: galaxies that emit strong radio waves.

In the active galactic nucleus (AGN), optical variability is an important index to measure the time-varying radiation flux of objects. The analysis of optical variability is a common method to study the radiation mechanism and internal structure and its change of objects. Some useful physical parameters, such as optical variability period, can be obtained by analyzing the time series of the optical variability of objects. For example, the known period can be used to estimate the mass of objects and the factors driving the periodic optical variability, the radiation mechanism of the active galactic nucleus, the energy mechanism of the center and the physical properties of the central nucleus.

Blazar is a class of active galactic nuclei that attracts much attention. According to the observed phenomena, Blazar can be divided into two important subclasses: BL Lac and FSRQ. Jurkevich method, wavelet analysis, power spectrum method, period04 method, discrete correlation function, DCDFT and so on are used to study optical the variability period of Blazar, among which Jurkevich method is more commonly used. 


\section{Jurkevich Method and Application}

Jurkevich method is a periodic method based on the mean square error of the expected value, which was proposed by Jurkevich on the basis of non-uniform observations in 1971. Because of the convenience, Jurkevich method has been widely used, especially in the study of the optical variability of Blazar objects. Next, we will summarize the application of Jurkevich method in astrophysics research.

Xiong Zhang, Guangzhong Xie and Jingming Bai ${ }^{[1]}$ used Jurkevich method to analyze the historic optical variability curves of 1642 effective data points of optical B-band of Quasar 3C 345 from 1896 to 1939. It was found that Quasar 3C 345 has $10.1 \pm 0.8$ years and $21.8 \pm 1.5$ years of period and the latter is twice of the former. So it belongs to one cycle. The results showed that Jurkevich method is more sensitive, accurate and effective for long period analysis. Chengyue $\mathrm{Su}^{[2]}$ used the same method to study and analyze B-band of PKS $2251+158$. It was found that there is a 12.39 years of quasi periodic optical variation. The results again proved that Julkevich method is very good for dealing with periodic problems. Kaibo Liu, Dongmo Yang and Dedong Hou et al.[3] used Jurkevich method to analyze B-band optical properties of 3C 120 celestial body in Application of Jurkevich method to the analysis of period of 3C 120 celestial body. By comparing with other studies of the period of 3C 345 quasar, Jurkevich method is considered to be an effective and accurate method to search for the optical variability period of 3C 120 currently, which is very useful for long-term monitoring of periodic analysis of objects and objects.

In the Analysis of periodic characteristics of quasar 3C 273 X-ray band ${ }^{[4]}$, Guangliang Qiu, Hongjun Ma and Xiong Zhang et al. also used DCF method and Jurkevich method to study and analyze the historic optical variability curves of QSO 3C $273 \mathrm{X}$-ray band (2-10 KeV) from 1976 to 2008 from 1059 observation data points. The results of the two periodic methods are the same. In the Long-term period Analysis of BL Lac Object AO 0235+164, Shengle Wen ${ }^{[5]}$ used Jurkevich and other methods to analyze the optical B-band data of the AO $0235+164$. The results were verified by DCF method, Montel Carlo method and folding method. It was found that the period of AO $0235+164$ may be 1.56 years.

Xiongwei $\mathrm{Bi}$, Wanquan $\mathrm{He}$, Jiajin Tian et al. ${ }^{[6]}$ used discrete correlation function method, Jurkevich method and power spectrum method analyze the curves periodic change of BL Lac celestial body Mrk 421 in 15-50 keV X-ray band from February 12, 2005 to November 26, 2012. It is concluded that 1.7 years is the inherent periodic component of Mrk 421 in X-ray band. On the other hand, it is found that the fitting periodic curve is basically consistent with the observed data. On the basis of previous observations, the author studied and analyzed the data obtained, and thus obtained more detailed information about BL Lac object Mrk 421, which is conducive to future astronomical research.

\section{Conclusion}

In summary, optical variability analysis is a common method to study the radiation mechanism and internal structure of objects and their changes. By observing the optical variability behavior of objects, we can analyze their optical variability characteristics, estimate the mass of the central black hole of objects, and study the causes of periodic optical variability of objects. The credibility of the method is very high. In this paper, we synthesized six papers on the analysis of the optical variability period of objects using Jurkevich method. In the papers published byXiong Zhang and others, Jurkevich method, DCF method and wavelet analysis method are used to analyze the optical variability period of objects respectively. The results show that the conclusions are basically consistent. In the papers published by Xiongwei Bi and others, Jurkevich method, power spectrum method and discrete correlation function method are used to study and analyze the optical variability of objects, and the conclusions obtained by different methods are similar. There are other researchers who use Jurkevich method and compare and analyze other optical variability period analysis methods, the conclusions are also the same. The above results show that Jurkevich method is a more commonly used method for studying optical variability period of active galactic nuclei. 
This method is easy to operate and requires little observation data. It can analyze and process the non-uniform data, and the analysis results are in good agreement with the experimental results. Therefore, Jurkevich method can be used to analyze the observed objects more accurately, improve the observation efficiency, and make a prediction for enriching the periodic optical variability data of the active galactic nucleus; Jurkevich method used in the analysis of optical variability can also explain the movement of the target objects, star structure and characteristics and its theoretical models, which can provide convenient conditions for astrophysical research.

\section{Acknowledgements}

This work is supported by the Science Research Project of Education Department of Yunnan Province, China(2018JS506).

\section{References}

[1] Zhang Xiong, Xie Guangzhong, Bai Jinming. Study on the Characteristics of Photoperiod of Quasar 3C 345[J]. Journal of Astronomy, 1998,39(3), 256-264.

[2] Su Chengyue. Long-term Optical Variational Period Analysis of Quasar PKS 2251+158[J]. Astrophysical Journal, 2000,12-16.

[3] Liu Kaibo, Yang Dongmo, Hou Dedong, Zhang Xiong, Ding Liang, Tang Ling, Dong Futong. The Application of Jurkevich in period Analysis of 3C 120 objects [J]. Journal of Yunnan Normal University, 2009, 1-6.

[4] Qiu Guangliang, Ma Hongjun, Zhang Xiong, Zheng Yonggang. Analysis of X-ray Band Periodicity Characteristics of Quasar 3C 273 [J]. Journal of Yunnan Normal University, 2012, 32 (3), 12-16.

[5] Wen Shengle. Long-term period Analysis of BL Lac Object AO 0235+164[J]. Journal of Natural Science, Hunan Normal University, 2004,27(2), 45-47.

[6] Bi Xiongwei, He Wanquan, Tian Jiajin, Cai Qun, Ding Zhimei, Zhang Qingyou. Study on the Variation Characteristics of X-ray Radiation Flow of BLLac Object Mrk 421 [J]. Astronomical Research and Technology, 2013, 10(3), 207-213. 